\title{
Ampliando a usabilidade de interfaces web para idosos em dispositivos móveis: uma proposta utilizando design responsivo
}

\author{
Afonso Alban \\ Curso de Ciência da Computação - Universidade de Passo Fundo - \\ afonsoalban@gmail.com \\ Ana Carolina Bertoletti De Marchi \\ Mestrado em Envelhecimento Humano - Universidade de Passo Fundo - \\ carolina@upf.br \\ Silvana Alba Scortegagna \\ Mestrado em Envelhecimento Humano - Universidade de Passo Fundo - \\ silvanalba@upf.br \\ Camila Pereira Leguisamo \\ Mestrado em Envelhecimento Humano - Universidade de Passo Fundo - \\ camila@upf.br
}

\begin{abstract}
This paper presents a proposal to extend the usability of web interface for elderly people in mobile devices using responsive web design. From a web interface implementation simulations were performed at different screen sizes. The simulations met expectations, once the technique allows only one content and formatting it according to the specifications of each output, besides creating mechanisms for easy learning of data entry. Such mechanisms are important to minimize usability problems for the elderly, caused by the small size of the screens and fields for data entry, often difficult to visualize.
\end{abstract}

Resumo. Este artigo apresenta uma proposta para ampliar a usabilidade de interfaces web para idosos em dispositivos móveis com o uso de design responsivo. A partir da implementação de uma interface web foram realizadas simulações em diferentes tamanhos de telas. As simulações atenderam as expectativas, pois a técnica possibilita produzir somente um conteúdo e formatá-lo conforme as especificações de cada saída, além de criar mecanismos de fácil aprendizagem para a entrada de dados. Tais mecanismos são importantes para minimizar problemas de usabilidade dos idosos, acarretados pelo tamanho reduzido das telas e dos campos para entrada de dados, muitas vezes de difícil visualização.

\section{Introdução}

Há algum tempo, pesquisas relevam o aumento da expectativa de vida da população, devido às melhorias na qualidade de vida. Dados recentes publicados no 
relatório da Organização das Nações Unidas apontam que até 2050 a população idosa deve chegar a 2 bilhões. No Brasil a estimativa é de 64 milhões, o triplo dos dias atuais.

Diretamente proporcional ao envelhecimento populacional, encontra-se o aumento no uso da Internet por idosos. Em pesquisa realizada pelo Pew Research Center's Internet \& American Life Project (Zickuhr e Madden, 2012) dados apontam que $53 \%$ de adultos americanos com idade igual ou superior a 65 anos usam internet ou email, representando um aumento de quase $200 \%$ se comparado a 2002.

$\mathrm{O}$ acesso a Internet por idosos não se dá apenas por meio de computadores. No Brasil, 37\% dos adultos com mais de 60 anos navegam na Web com dispositivos móveis, frente a $29 \%$ de jovens, conforme pesquisa do Instituto QualiBest. Verifica-se que, especialmente para os idosos, a inclusão digital atinge várias esferas, promove o incremento da inserção social, afetiva e consequentemente da saúde mental, uma vez que, conectar-se com pessoas e com o conhecimento minimiza o impacto das perdas e transformações que incidem nesta etapa.

Navegar pela Web apresenta-se como uma resposta inovadora aos novos desafios e demandas sociais, sendo a aprendizagem contínua a sinalizadora da possibilidade pedagógica para os indivíduos idosos como consolidação de troca e aquisição de conhecimentos e socialização (Marinho, 2007). O idoso passa a dar lugar a novos conhecimentos, técnicas e reformular seus saberes.

Estar motivado e satisfeito incrementa a capacidade de raciocinar, compreender adequadamente a realidade, e de manter uma atitude crítica. Quando os idosos despendem seu tempo com atividades gratificantes, adaptam-se melhor ao envelhecimento. Muito embora, o envelhecimento acarrete diversas mudanças no ser humano, envolvendo redução da acuidade visual e cognitiva, lentidão nas ações de reação e do raciocínio, dentre outras que refletem diretamente na interação com o computador e dispositivos móveis.

Sendo assim, projetar interfaces para terceira idade abrangendo diferentes dispositivos exige um cuidado extra com a organização das informações, facilidade de interação, adaptação a diferentes ambientes, entre outros aspectos relacionados ao design, a web e a usabilidade, mostrando-se necessária a identificação de diretrizes de usabilidade específicas.

Paralelamente ao crescimento de idosos, cresce também o acesso à internet por meio de dispositivos móveis, em decorrência eleva-se a oferta de dispositivos com diferentes características: resolução, tamanho e formato da tela, formas de entrada de informação, processamento, memória, suporte à plug-ins etc.; além das já conhecidas restrições do ambiente sem fio (Bonifácio et al., 2010). Pensar as alterações próprias do envelhecimento frente aos diferentes formatos de telas, resoluções e sistemas tão distintos implica em estratégias que possam vir a favorecer a interação desse público.

Atualmente, mostra-se quase impossível criar versões específicas de cada website para computadores de mesa, tablets e smartphones; por isso a necessidade de se pensar em uma tecnologia que possibilite adaptar automaticamente - e da melhor forma possível - diferentes conteúdos em diferentes plataformas. Nesse contexto, cresceu nos últimos meses, na comunidade de designers de interface, o conceito de design responsivo (responsive web design) - o design que se ajusta a diferentes dimensões, resoluções, comportamentos de tela, plataformas e usuários.

Diante do exposto, este trabalho apresenta uma proposta para ampliar a usabilidade de interfaces web para idosos em dispositivos móveis por meio do uso do design responsivo. As simulações, realizadas em diferentes tamanhos de telas e levando 
em consideração algumas convenções apontadas na literatura, atenderam as expectativas quanto à disposição do conteúdo, elementos de entrada de dados e navegação.

\section{Usabilidade de Interfaces Web para Idosos em Dispositivos Móveis}

Interface é a parte através da qual o usuário interage com o sistema, muitas vezes considerando-a o próprio sistema (Hix e Hartson, 1993). Com o passar do tempo e a evolução da tecnologia, as interfaces foram se modificando, novas formas de interação surgiram e outras caíram em desuso, até chegarmos à interface gráfica e, mais recentemente, a interface de toque na tela, popularizada nos smartphones e tablets.

Estritamente ligada às interfaces está a questão da usabilidade: um atributo de qualidade relacionado à facilidade de uso de algo (Nielsen, 2007). A usabilidade permite avaliar a interface segundo à curva de aprendizado, eficiência e facilidade de uso, satisfação, entre outros fatores.

Com a possibilidade de conexão em praticamente qualquer lugar habitado hoje, cresce o número de pesquisas e investimentos na usabilidade de interfaces de dispositivos móveis, de modo a tentar transportar o poder computacional presente em computadores "de mesa" para dispositivos de fácil mobilidade (RIBEIRO, 2007).

Frente às demais plataformas, podemos apontar dois problemas ao se projetar interfaces móveis para idosos que dificultam sua inclusão digital, a saber:

i)problemas técnicos: ocasionado pela variedade de modelos de aparelhos e, consequentemente, diferentes tamanhos de tela e quantidades de pixels apresentados; interação através de um teclado físico ou por toque na tela, e ainda o suporte a gestos com dois dedos ou mais; suporte a plug-ins; disponibilidade de rede; poder de processamento e memória etc.

ii)problemas de interação: resultado do processo de envelhecimento que afetam aspectos físicos e cognitivos como, por exemplo, a redução da visão, da precisão de movimentos e da memória.

A pesquisa de Tang e Kao (2005) objetivou compreender os problemas enfrentados pelos idosos durante a utilização de telefones celulares, fazendo referência as mudanças demográficas da população; ao fato dos idosos terem se tornado um grande grupo da sociedade e a concentração das indústrias de dispositivos móveis em adolescentes. Os autores aplicaram um teste de usabilidade a 39 idosos para analisar as operações nos telefones móveis. Todos os sujeitos com experiência de 1 a 5 anos na utilização de telefones móveis. Os resultados foram apresentados em 4 níveis: físico, perceptual, funcional e conceitual, baseado no modelo conceitual de Norman. Dentre os problemas apontados que prejudicam a usabilidade para idosos estão: tamanhos pequenos de botões; botões sem rótulos; compreensão dos ícones e estrutura hierárquica de menus.

A maioria dos projetistas de websites é jovem e, muitas vezes, assumem que todos os usuários possuem uma visão e controle motor perfeitos. Para Nielsen (2002), dentre os principais problemas de interação dos idosos estão: i) fontes com tamanhos reduzidos; ii) menus pull-down, que surgem quando o mouse passa em cima de um determinado elemento; iii) dificuldade em utilizar mecanismos de busca; iv) não distinção de cores para links visitados e v) mensagens de erro de difícil leitura.

Especialmente em dispositivos móveis, existem barreiras que restringem ainda mais seu uso por idosos como, por exemplo, o tamanho reduzido das telas para melhor propiciar a mobilidade; os campos para entrada de dados, muitas vezes de difícil 
visualização e a grande diversidade de menus que dificultam a memorização. Ainda, se o dispositivo móvel é utilizado para acesso de páginas web, o agravante reside no fato de que grande parte dos websites não possui versão para telas menores, tornando a visualização e a interação complexa dada a exigência do uso das barras de rolagem horizontal e vertical. Imagens, campos de busca e de entrada de dados e conteúdo ficam distribuídos de forma desproporcional, afetando a usabilidade e, muitas vezes, exigindo compensações corporais na busca da adaptação e acarretando acometimentos físicos. Neste sentido, boas práticas para o desenvolvimento de interfaces para dispositivos móveis fazem-se necessárias, de modo a facilitar o projeto com o estabelecimento de orientações básicas.

\section{Estabelecendo boas práticas para dispositivos móveis}

Apesar das interfaces móveis não serem tão recentes, apenas nos últimos anos foi possível estabelecer orientações para designs deste tipo. Muitos dos princípios permanecem os mesmos utilizados na "web tradicional", porém, com o tempo, novas particularidades serão identificadas e soluções melhores surgirão para o design em dispositivos móveis, especialmente para idosos. Para um dispositivo onde o espaço de exibição é mínimo, a conexão é lenta e a interação é difícil, a regra unânime entre os profissionais e estudiosos da área é: foco no conteúdo realmente relevante, pelo menos no começo.

Ma (2011), aborda dez fatores que devem ser pensados de maneira diferente, quando se projeta um website para plataformas móveis. Entre eles:

a)priorização de conteúdo: enquanto websites projetados para computadores de mesa possuem um conteúdo vasto e detalhado, os websites mobile devem focar no conteúdo e nas funcionalidades mais essenciais inclusive levando em conta o local onde está o usuário. É preciso repensar a organização da informação e definir qual conteúdo o usuário vai precisar quando estiver acessando por um dispositivo móvel, inclusive diminuindo a hierarquia estrutural das páginas. O Google Maps mobile, por exemplo, identifica a posição do usuário e apresenta resultados relevantes na área em que o usuário está. Na versão padrão é necessário selecionar um local antes, para então efetuar a busca naquele mapa.

b)navegação vertical: sites desktop possuem, evidentemente, mais espaço para a apresentação de uma navegação horizontal. Porém, é comum que versões mobile apresentem os menus verticalmente, facilitando a visualização e a navegação para o usuário.

c)links: formatar links para que se pareçam com links é uma prática aconselhada para qualquer website. Em desktops isso geralmente é feito alterando a cor do link, o tipo da fonte ou sublinhando o texto - o que é facilmente clicado com um dispositivo apontador. Já em websites móveis, deve-se pensar em uma maneira que facilite o clique através dos dedos; por isso, devem ter uma área clicável maior, e se assemelharem a botões, ajudando o usuário a identificar o link.

d)imagens: se em websites desktops as imagens são usadas para os mais diversos fins (ilustrações, infográficos, propagandas etc.), em versões móveis deve ser priorizado o texto "puro", pois, além dos tamanhos de 
tela muito divergentes (o que vai mudar a forma como o usuário visualiza a página), em muitos locais a internet móvel ainda é lenta.

e)navegação local: é comum remover a navegação local em dispositivos móveis, deixando apenas a navegação global. Isso está extremamente ligado à priorização de conteúdo e a reorganização da informação no website.

f)breadcrumbs: se um site está organizado de maneira simples, não há a necessidade de rastrear e exibir o caminho da página na hierarquia do site, uma vez que o usuário dificilmente ficará perdido.

g)integração com funções do dispositivo: apesar das várias restrições que os dispositivos móveis apresentam, existem novas possibilidades de comunicação através deles. Sites podem tirar proveito de funções como ligações ou mensagens de texto, e facilitar a execução dessas ações para o usuário.

O W3C também propôs algumas orientações para websites móveis, como i) levar em conta diferentes dispositivos; ii) utilizar de padrões web em marcação, disponibilização e formatação do conteúdo; iii) evitar o uso de tabelas aninhadas, janelas pop-ups, frames e imagens mapeadas; iv) organizar o documento de forma que seja possível entendê-lo sem folhas de estilo; v) navegação simples; vi) URLs curtas; vii) não utilizar medidas em pixels ou em unidades absolutas; viii) fornecer informações para uso eficiente de cache nas chamadas HTTP; ix) evitar a entrada de texto; $x$ ) rotular todos os controles de formulário de maneira adequada (W3C, 2008).

As orientações propostas pelo $\mathrm{W} 3 \mathrm{C}$ atendem as principais dificuldades apontadas na interação de idosos com dispositivos móveis, como, por exemplo, a dificuldade com janelas pop-ups.

\section{Design responsivo}

Atualmente, ao se projetar um website é comum levar em consideração o desenvolvimento de uma versão para acesso móvel. Como se já não bastassem as várias resoluções de tela de dispositivos desktop, existem ainda os diferentes tamanhos (e características) de variados dispositivos móveis, o que torna impraticável construir websites específicos para cada plataforma e aparelho, seja pelos inúmeros modelos no mercado, seja pelo alto custo desta política de desenvolvimento.

Nesse contexto, tem-se a necessidade de buscar uma solução que compreenda e se adapte ao comportamento do usuário e ao ambiente que ele está usando para acessar as informações, levando em conta resolução de tela, a plataforma e, até mesmo, a orientação da tela. $\mathrm{O}$ design responsivo procura suprir essa necessidade: utilizando um misto de grids, imagens, marcações CSS e, possivelmente, scripts, possibilita ao website a habilidade de "responder" às preferências do usuário (Knight, 2011). Porém, o desing responsivo vai além de apenas mostrar o website inteiro na tela, redimensionando a página e as imagens; ele propõe maneiras de apresentar o mesmo conteúdo em diferentes plataformas.

Segundo Doyle (2011), um design responsivo deve: i) adaptar o layout a diferentes tamanhos de tela; ii) redimensionar imagens conforme a resolução da tela; iii) fornecer imagens otimizadas para banda estreita, para dispositivos móveis; iv) simplificar elementos da página para versões móveis; v) ocultar elementos menos importantes em telas menores; vi) fornecer botões e links com grande área clicável para 
dispositivos móveis; vii) detectar (e utilizar, se necessário) funcionalidades específicas dos dispositivos móveis, como geolocalização e orientação da tela.

Segundo Marcotte (2011), um design responsivo só pode ser criado utilizando-se três elementos básicos: i) layout flexíveis, baseados em grades; ii) imagens e outras mídias (como áudio e vídeo) visualmente flexíveis; e iii) media queries.

Layouts flexíveis consistem em páginas projetadas para se auto-ajustarem a qualquer resolução de tela ou tamanho da janela do navegador, mantendo a distribuição proporcional ao tamanho originalmente projetado. Para isso, são utilizadas grades - ou grids - que ajudam a definir a posição e os valores corretos matematicamente. Geralmente, um layout é desenhado utilizando-se medidas absolutas em pixels; num segundo momento, este é convertido para medidas relativas - em valores percentuais mantendo as proporções originais.

Imagens flexíveis se ajustam proporcionalmente ao espaço aonde estão inseridas - os containers. Como o design responsivo está sendo desenvolvido visando seu funcionamento em browsers modernos, com suporte a HTML5 e CSS3, é importante ressaltar que outros tipos de mídia - como vídeo e áudio (visualmente representado por uma barra de progresso) - também devem seguir o mesmo comportamento das imagens.

Consultas de mídia, conhecidas pelo nome em inglês "media queries" são uma evolução do atributo media type (tipos de mídia), concebido como parte da especificação CSS2, e amplamente utilizados para definir folhas de estilo específicas para tela ou impressora - com cores e tamanho da fonte específicos para cada saída, por exemplo. Os valores aceitos variam conforme os tipos de mídia especificados (podem ser valores "all", "screen", "print", "speech", "projection" etc.). Como a definição do tipo de saída de um documento não é mais suficiente - o valor "tela" se tornou amplo demais -, o CSS3 traz as consultas de mídia, permitindo consultar não só a mídia, mas também as características físicas do dispositivo e do browser que exibe o conteúdo (Marcotte, 2011). Esta é a principal característica que permite o funcionamento do desing responsivo; apenas com as media queries, especificadas nos documentos do CSS3, é possível descrever características especiais para determinadas configurações.

Somente um layout fluido com imagens proporcionalmente dimensionadas não será eficiente sem o uso de media queries, pois é este mecanismo que permite reorganizar o conteúdo conforme o espaço disponível para tal. Sendo assim, as características que podem ser consultadas variam desde a largura e altura do navegador e do browser, a orientação do dispositivo (se a interface está na vertical ou na horizontal); até o aspect-ratio da tela, a densidade de pixels e a quantidade de cores suportadas pelo dispositivo.

Aos três itens apontados por Marcotte, este trabalho propõe um quarto elemento: o uso de recursos de interface do próprio dispositivo para auxiliar a interação do idoso. Atualmente, os sistemas operacionais para dispositivos estão suficientemente desenvolvidos e possuem recursos que auxiliam na execução de determinadas tarefas ao navegar pela web. No iOS, por exemplo, um campo de entrada de um formulário marcado como tipo "number" exibe na tela um teclado composto apenas por números em contraste ao tipo "text" que apresenta um teclado alfanumérico padrão.

\section{Protótipos}

Para verificar a eficiência da técnica no desenvolvimento de interfaces multiplataformas foram desenvolvidos três protótipos que exemplificam casos comuns à maioria dos websites e, ainda assim, distintos. Para facilitar o processo foi utilizado um 
boilerplate chamado 320 and Up. O conjunto de arquivos foi escrito e organizado por Andy Clarke, como uma extensão do HTML5 Boilerplate - um framework para desenvolvimento de interfaces em HTML5.

O boilerplate foi organizado visando a construção da página a partir dos dispositivos menores - tela com 320 pixels de largura. $\grave{A}$ folha de estilos base são adicionados outros arquivos que a incrementam com diferentes funcionalidades (funções, classes predefinidas, variáveis e operações que definem medidas e cores, tipografia etc.), até chegar às folhas correspondentes ao layout da página em telas maiores. Por se tratar de um processo incremental, cada nova folha adiciona "características" específicas a determinado caso.

Os protótipos foram desenvolvidos em HTML5 e CSS3/Less, codificados em um computador rodando Mac OS X Lion, com resolução de 1280x800 pixels. Posteriormente, foram realizados testes em computadores rodando Windows, em diversas resoluções $(1400 \times 900,1024 \times 768,1280 \times 800)$; iPhone $4 \mathrm{~S}$ rodando iOS 5, com resolução de 960x640, a 326 pixels por polegada - corresponde a uma tela de 480x320 pixels; iPad 2 rodando iOS 5, com resolução de 1024px por 768px; diferentes celulares rodando Android, entre eles um Samsung Galaxy Y, com resolução 240x320 pixels, com Android 2.3 Gingerbread.

Nas próximas seções, apresentamos detalhes acerca dos três protótipos desenvolvidos: um modelo de página inicial, um modelo de artigo e um modelo de formulário.

\subsection{Página Inicial}

O primeiro teste foi sobre uma página inicial. Considerando as telas menores, o tamanho da fonte e a disposição do conteúdo foram adaptados para facilitar a leitura e a interação: sem usar design responsivo, no smartphone o usuário precisa utilizar movimentos de zoom e rolar a página para todos os lados para conseguir ler o conteúdo (Figura 1). No protótipo, o texto apresenta um tamanho agradável à leitura e a rolagem acontece apenas verticalmente - tanto no smartphone, quanto no desktop (Figura 2).

Nas telas menores, a característica slider do menu horizontal é indicada por setas; e o rodapé é reduzido para mostrar apenas as informações julgadas relevantes àquele ambiente - aqui é aplicado o conceito da priorização do conteúdo. As imagens se adaptam ao espaço disponível, bem como o texto e o tamanho da fonte, facilitando a leitura.

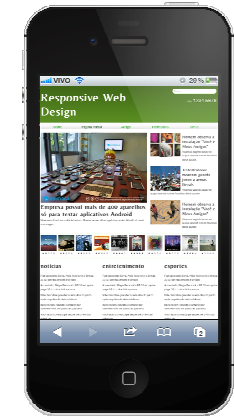

Figura 1 - Layout feito sem uso de técnica

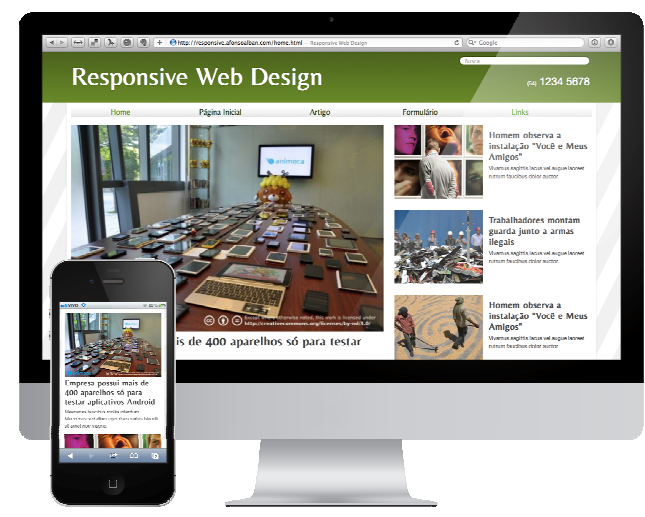

Figura 2 - Design responsivo em diferentes dispositivos 


\subsection{Artigo}

A página que representa um artigo é dividida numa coluna principal (onde está o texto), e outra lateral (onde estão links relacionados). Nas telas menores, há apenas uma coluna; as imagens preenchem toda a tela, e os trechos de texto são posicionados acima e abaixo - nunca ao lado (Figura 3).

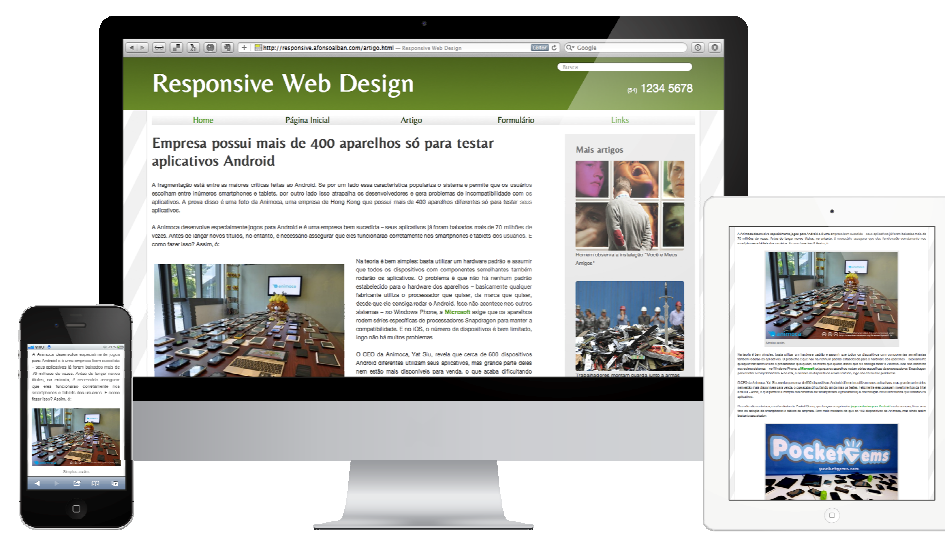

Figura 3 - 0 texto do artigo se ajusta ao espaço disponível, sem prejudicar a exibição das imagens

Este protótipo foi codificado usando diversos elementos novos do HTML5, como article para marcar a área do texto principal, e aside para marcar o conteúdo relacionado. Essa marcação, além de facilitar a classificação do conteúdo na web, permite que, no futuro, os dispositivos incluam formatação adicional a pedaços específicos da página. No final do artigo, está inserido um vídeo - este também se ajusta ao tamanho da página. Porém, a técnica não permite que sejam carregados vídeos de diferentes resoluções conforme o dispositivo - para isso é necessário, no mínimo, um script no cliente ou no servidor.

Em telas menores, a coluna lateral é coloca abaixo do artigo principal, e as imagens são removidas, tornando-a uma lista simples de links.

\subsection{Formulário}

A entrada de dados é um dos maiores problemas de usabilidade, principalmente em idosos: dados incompletos, informações incoerentes com o campo onde foram digitadas, campos obrigatórios não preenchidos etc. No ambiente móvel, os dispositivos são preparados para facilitar essa entrada, ajudando a reduzir erros comuns - porém, há a necessidade de que esse recurso seja explicitamente marcado no código HTML, utilizando-se tipos de entrada especiais.

As entradas especiais apresentadas nos documentos do HTML5 são várias apenas algumas foram utilizadas neste trabalho. Os diferentes tipos de entrada de texto habilitam teclados específicos para cada informação. O tipo text veio de versões anteriores do HTML, e possibilita digitar qualquer tipo de texto. $\mathrm{O}$ tipo email obriga o usuário a inserir um endereço de email válido - o teclado do dispositivo auxilia o usuário, fornecendo atalho para a “@”, por exemplo. O tipo tel especifica a entrada de um telefone - o teclado apresenta apenas números, dispostos como um teclado de telefone padrão. Por fim, o tipo date espera a entrada de uma data válida - o sistema facilita o processo apresentando as opções disponíveis de maneira intuitiva (Figura 4). Cabe ressaltar que estas melhorias na interface ocorreram apenas em dispositivos 
móveis; nos navegadores para desktop, os dados entrados apenas são validados de acordo com o que se espera.

Uma das propostas deste trabalho é, justamente, orientar para que se faça uso desses novos tipos de entrada de dados, a fim de facilitar a interação do idoso com a interface. Um design responsivo deve integrar-se com funções do dispositivo, quando possível. Neste sentido, no iPhone, por exemplo, também é possível realizar uma ligação tocando no número de telefone marcado como tal.
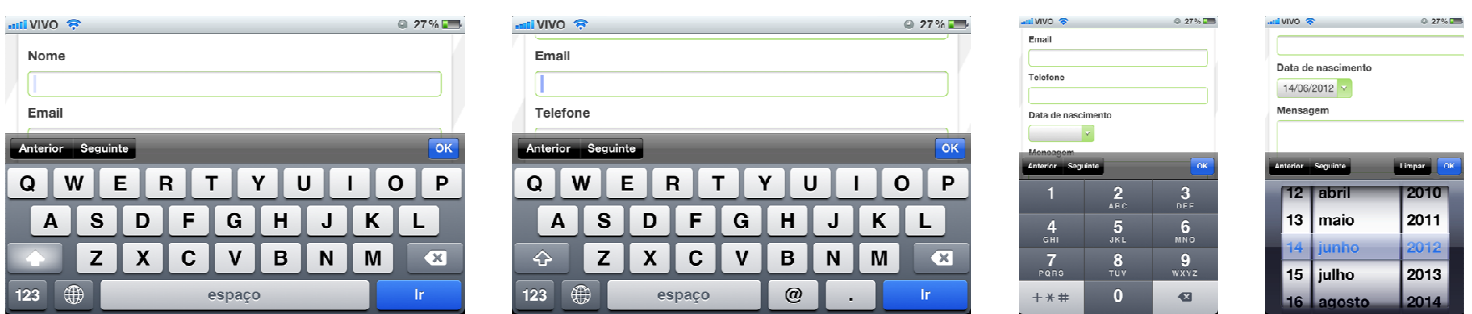

Figura 4 - Diferentes layouts de teclado para diferentes informações

\section{Conclusão}

Como alternativa para atender a crescente demanda de usuários idosos se incluindo digitalmente por meio da interação com websites móveis, a técnica de design responsivo se mostra satisfatória, pois possibilita produzir somente um conteúdo, e formatá-lo conforme as especificações de cada saída, além de criar mecanismos de fácil aprendizagem para a entrada de dados. Para que isso ocorra, portanto, é preciso fazer uso de layouts e imagens fluidos, e media queries. Junto a esses requisitos, a experiência do usuário pode ser melhorada fazendo uso de novos recursos de HTML5, e tirando proveito, quando possível, das funções de cada dispositivo.

Por se tratar de uma técnica nova, grande parte da comunidade de desenvolvedores desconhece as maneiras eficientes de aplicação. Neste sentido, o uso de um boilerplate facilita e agiliza o processo, pois proporciona ao desenvolvedor iniciar formatando uma interface simples e, em incrementos, adicionar estilos que satisfaçam a experiência em diversos dispositivos.

Como trabalho futuro, a técnica poderá ser validada com um grupo de idosos para atestar seu benefício na melhora da usabilidade e inclusão digital, como também, para contribuir com o incremento da qualidade de vida desta população que cresce.

\section{Referências}

BONIFÁCIO, Bruno et al. Aplicando Técnicas de Inspeção de Usabilidade para

Avaliar Aplicações Móveis. Departamento de Ciência da Computação. Universidade

Federal do Amazonas, Manaus, 2011. Disponível em:

<http://www.dcc.ufam.edu.br/uses/index.php/publicacoes/doc_download/85-ihc-

2010aplicando-tecnicas-de-inspecao-de-usabilidade-para-avaliar-aplicacoesmoveis>.

CLARKE, Andy. This is the new 320 and up. In: Stuff \& Nonsense, 20 abr. 2012.

Disponível em:

<http://www.stuffandnonsense.co.uk/blog/about/this_is_the_new_320_and_up>. Acesso em: 23 mai. 2012.

DOYLE, Matt. Responsive Web Design Demystified. In: Elated, 30 set. 2011.

Disponível em: <http://www.elated.com/articles/responsive-web-designdemystified>. 
HIX, D.; HARTSON, H.R. Developing user interfaces: ensuring usability through product \& process. New York: John Wiley, 1993. 381p.

KNIGHT, Kayla. Responsive Design: What It Is and How to Use It. In: Smashing Magazine, 12 jan. 2011. Disponível em:

<http://coding.smashingmagazine.com/2011/01/12/guidelines-for-responsive-webdesign>. Acesso em: 27 mai. 2012.

MA, Shanshan. 10 Ways Mobile Sites Are Different from Desktop Web Sites. In: UX Matters, 21 mar. 2011. Disponível em:

<http://www.uxmatters.com/mt/archives/2011/03/10-ways-mobile-sites-aredifferent-from-desktop-web-sites.php>. Acesso em: 16 nov. 2011.

MARCOTTE, Ethan. Responsive web design. A Book Apart: New York, 2011.

MARINHO, S. C. C.; Educação para idosos: um caminho para cidadania. In: ANAIS SIMPÓSIO BRASILEIRO DE POLÍTICA E ADMINISTRAÇÃO DA

EDUCAÇÃO, 23, 2007, Porto Alegre. Porto Alegre: ANPAE, 2007.

NIELSEN, Jakob. Designing Web Usability: The Practice of Simplicity. New Riders: Indianapolis, 1999.

NIELSEN, Jakob; LORANGER, Hoa. Usabilidade na web: Projetando Websites com qualidade. Rio de Janeiro: Elsevier, 2007.

RIBEIRO, Daniel F. Estudo de Interface Humano-Máquina em Dispositivos Móveis. Departamento de Informática e Estatística. Universidade Federal de Santa Catarina, Florianópolis, 2007. Disponível em: <http://projetos.inf.ufsc.br/arquivos_projetos/projeto_521/ArtigoDaniel.pdf>.

TANG, H. H.; KAO, S.A. Understanding the Real Need of the Elderly People When Using Mobile Phone, HCII 2005. Las Vegas. 2005.

W3C. Boas práticas em web móvel. In: World Wide Web Consortium - Mobile Web Initiative, [2008?]. Disponível em: <http://www.w3.org/TR/mobile-bp>. Acesso em: 3 mar. 2012. 